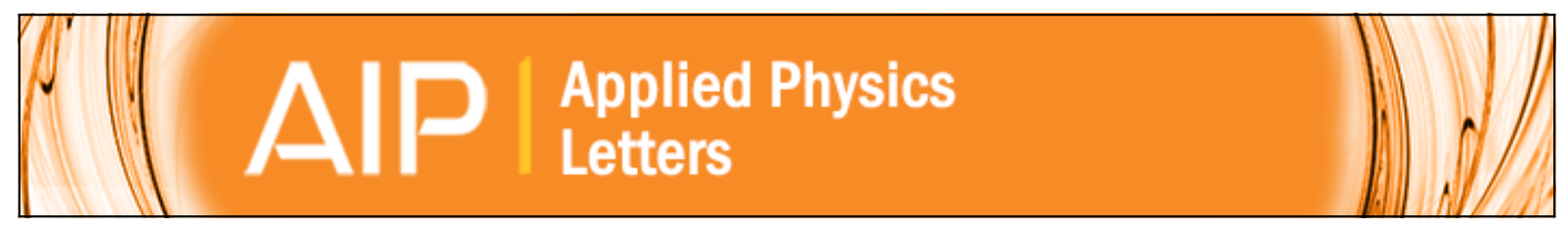

\title{
Ab initio study of the effect of water adsorption on the carbon nanotube field-effect transistor
}

Dongchul Sung, Suklyun Hong, Yong-Hoon Kim, Noejung Park, Sanghyeob Kim, Sung Lyul Maeng, and Ki-Chul Kim

Citation: Applied Physics Letters 89, 243110 (2006); doi: 10.1063/1.2397543

View online: http://dx.doi.org/10.1063/1.2397543

View Table of Contents: http://scitation.aip.org/content/aip/journal/apl/89/24?ver=pdfcov

Published by the AIP Publishing

\section{Articles you may be interested in}

Ab-initio calculations for a realistic sensor: A study of $\mathrm{CO}$ sensors based on nitrogen-rich carbon nanotubes AIP Advances 2, 032115 (2012); 10.1063/1.4739280

Conductance of individual channels in a carbon nanotube field-effect transistor studied by magnetic force microscopy

J. Appl. Phys. 106, 114315 (2009); 10.1063/1.3265435

Hysteretic transfer characteristics of double-walled and single-walled carbon nanotube field-effect transistors Appl. Phys. Lett. 91, 143118 (2007); 10.1063/1.2789789

Ab initio study of semiconducting carbon nanotubes adsorbed on the $\mathrm{Si}(100)$ surface: Diameter- and registrationdependent atomic configurations and electronic properties

J. Appl. Phys. 100, 124304 (2006); 10.1063/1.2400404

Adsorption-induced conversion of the carbon nanotube field effect transistor from ambipolar to unipolar behavior Appl. Phys. Lett. 86, 093105 (2005); 10.1063/1.1869548

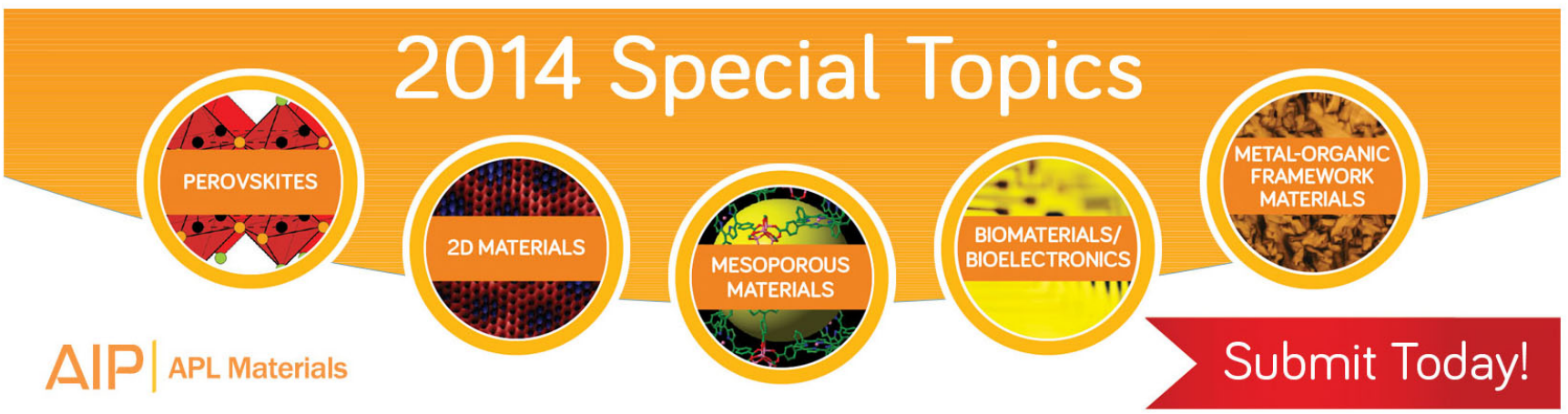




\title{
$A b$ initio study of the effect of water adsorption on the carbon nanotube field-effect transistor
}

\author{
Dongchul Sung and Suklyun Hong ${ }^{\text {a),b) }}$ \\ Department of Physics and Institute of Fundamental Physics, Sejong University, Seoul 143-747, Korea \\ Yong-Hoon Kim \\ School of Computational Sciences, Korea Institute for Advanced Study, Seoul 130-722, Korea \\ and Department of Materials Science and Engineering, University of Seoul, Seoul 130-743, Korea \\ Noejung Park ${ }^{\mathrm{a}), \mathrm{c})}$ \\ Department of Applied Physics, Dankook University, Seoul 140-714, Korea \\ Sanghyeob Kim and Sung Lyul Maeng \\ Cambridge-ETRI Joint R\&D Centre, Electronics and Telecommunications Research Institute, \\ Daejeon 305-700, Korea \\ Ki-Chul Kim \\ BK21 Physics Research Division, Institute of Basic Science, Sungkyunkwan University, Suwon 440-746, \\ Korea
}

(Received 14 August 2006; accepted 12 October 2006; published online 13 December 2006)

\begin{abstract}
We perform density-functional calculations to investigate the effect of adsorbed water molecules on carbon nanotubes (CNTs). Noting that the $\mathrm{H}_{2} \mathrm{O}$ molecule has much wider energy gap than the CNT, we find that the charge transfer between them is negligible. We discuss that several recent publications, which claimed a substantial electron transfer from the water molecule to the CNT, have been based on incautious interpretations of the Mulliken population analysis. We suggest that the effect of humidity on nanotube devices may be attributed to various indirect effects enhanced by water vapors, rather than the carrier generations by the physisorbed $\mathrm{H}_{2} \mathrm{O}$ molecules. (C) 2006 American Institute of Physics. [DOI: 10.1063/1.2397543]
\end{abstract}

Since the carbon nanotube field-effect transistor (CNTFET) was demonstrated experimentally in 1998, there has been widespread effort to understand the underlying physics as well as to improve the device performance. ${ }^{1-3}$ The characteristics of the CNTFET as a Schottky barrier transistor with a quasiballistic channel conduction may lead to an ultimately miniaturized electronic device. ${ }^{4,5}$ The potential of the CNTFET as a highly sensitive gas sensor has been demonstrated. ${ }^{6}$ The study of the adsorption effect on either the nanotube surface or metal-nanotube contact is still one of the most important subjects in the field. ${ }^{7-9}$ The effect of adsorption should be investigated in detail for the further development of the CNTFET as a gas sensor as well as a nanoscale electronic device. ${ }^{10-13}$

The effect of water or humidity on the CNTFET performance has been investigated experimentally as well as theoretically, but still remains arguable. ${ }^{14}$ In the transfer character measurement of the CNTFET, the hysteresis in $I-V_{g}$ curves has been commonly observed. The charge trapping and detrapping in the adsorbed water molecules have been suggested as the underlying mechanism for such hystereses. ${ }^{15}$ In addition, several literatures claimed that a substantial amount of electron transfer from the adsorbed water molecules to the nanotube, inducing a measurable electron doping in the semiconducting nanotube. In the present work, however, we show that such conclusion of the charge transfer between the $\mathrm{H}_{2} \mathrm{O}$ molecule and the nanotube was based on the incorrect

\footnotetext{
a) Authors to whom correspondence should be addressed.

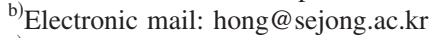

${ }^{c)}$ Electronic mail: noejung@dku.edu
}

interpretation of computational results. Instead, we suggest that the experimentally measured humidity effect should be ascribed to some indirect effects of the water vapors. As an example we show that the $\mathrm{OH}$ ions adsorbed on the $\mathrm{SiO}_{2}$ gate surface near the carbon nanotube generate trap levels, which could have substantial effect on the transport measurement.

For computation, we use the Vienna ab initio simulation package (VASP). ${ }^{16,17}$ The generalized gradient approximation (GGA) in the form of the PBE-type parametrization ${ }^{18}$ and the local density approximation (LDA) in the Ceperley-Alder form ${ }^{19}$ are employed. The ionic pseudopotentials are described via the projector augmented wave, ${ }^{20}$ and the cutoff energy for the plane-wave basis is set to $400 \mathrm{eV}$.

We first investigate the adsorption geometry of the $\mathrm{H}_{2} \mathrm{O}$ molecule on the outer wall of the isolated semiconducting nanotube. In this calculation, along the axial direction twice the minimal unit cell of the zigzag nanotube $(\approx 8.49 \AA)$ is chosen as the unit cell. In the perpendicular direction to the nanotube axis, the unit-cell dimensions are chosen such that the interatom distances in the neighboring cells are larger than $10 \AA$. We note that previous studies ${ }^{21,22}$ did not agree on the adsorption geometries of the water molecule on the carbon nanotube. In the present study, various adsorption sites, including the center of the hexagon, the bridge of the carboncarbon bond, and the top of the carbon atoms, are explored to determine the optimal adsorption geometry.

Geometry optimizations converged to several adsorption geometries with little energy difference. Their energies and geometries show that the adsorption of $\mathrm{H}_{2} \mathrm{O}$ is a mere physisorption. Two configurations with the lowest energies for the 


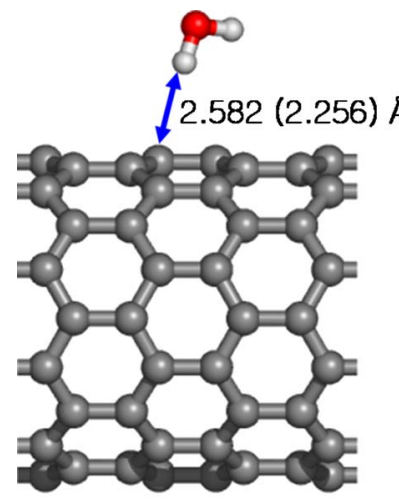

(a) $0.0 \mathrm{eV}$

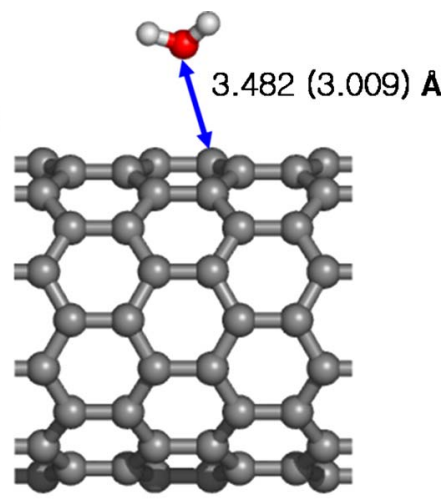

(b) $0.020(0.030) \mathrm{eV}$

FIG. 1. (Color online) Two optimized geometries for the $(10,0)$ carbon nanotubes with the adsorbed $\mathrm{H}_{2} \mathrm{O}$ molecule. The hydrogen atom is directed to the bridge of the $\mathrm{C}-\mathrm{C}$ bond in (a), and the oxygen atom is directed to the hexagonal center of the carbons in (b). The $\mathrm{C}-\mathrm{O}$ and $\mathrm{C}-\mathrm{H}$ distances and the total energy difference between the two geometries are shown. The results from the GGA and LDA calculations are written out of and in parentheses, respectively.

representative positions of water molecule are shown in Fig. 1. Between the optimized geometry where one hydrogen atom points toward the bridge of the $\mathrm{C}-\mathrm{C}$ bond [Fig. 1(a)] and that where the oxygen atom is more attracted toward the center of the carbon hexagon [Fig. 1(b)], both the LDA and GGA calculations find that the former geometry is slightly more stable. It is noteworthy that the PBE functional, which is used in the present work, has proved to be accurate in the hydrogen-bonded systems. ${ }^{23,24}$

The electronic band structure of pristine $(10,0)$ nanotube and that of the $\mathrm{H}_{2} \mathrm{O}$-adsorbed $(10,0)$ nanotube are shown in Figs. 2(a) and 2(b), respectively. We note that the band structure near the top of the valence bands or bottom of the conduction bands is negligibly changed within the computational accuracy upon the molecular $\mathrm{H}_{2} \mathrm{O}$ adsorption. The highest occupied molecular orbital (HOMO) level of $\mathrm{H}_{2} \mathrm{O}$ molecule is located about $2.5 \mathrm{eV}$ below the Fermi level of the carbon nanotube, as indicated by the blue leftward arrow in Fig. 2(b). The lowest unoccupied molecular orbital (LUMO) level of $\mathrm{H}_{2} \mathrm{O}$ molecule sits at about $4 \mathrm{eV}$ above the Fermi level, as denoted by the red arrow. We find that the LUMO-derived band is more or less dispersive. This should be attributed to the smallness of the supercell length, and the
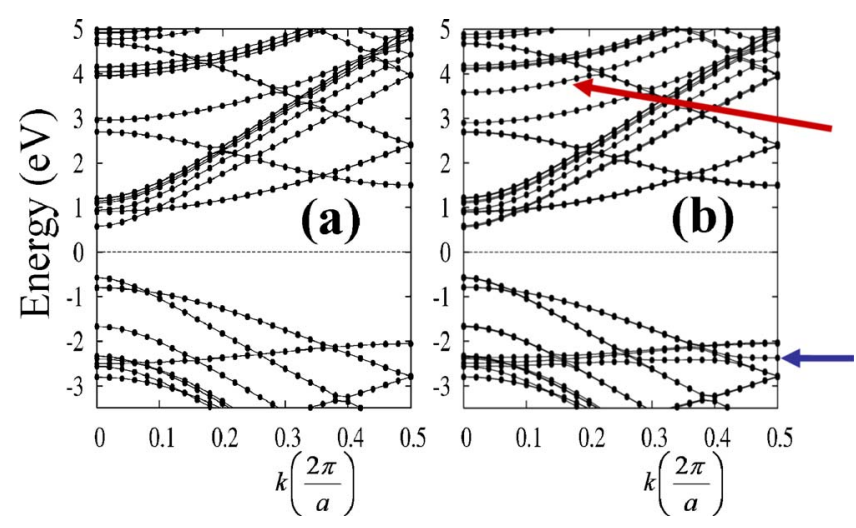

FIG. 2. (Color online) Electronic band structures for (a) the bare and (b) the $\mathrm{H}_{2} \mathrm{O}$-adsorbed $(10,0)$ carbon nanotubes. The blue short arrow and the red long arrow indicate the HOMO and LUMO states of $\mathrm{H}_{2} \mathrm{O}$ molecule respectively.

TABLE I. Charge of the water molecule adsorbed on CNT calculated by various basis sets.

\begin{tabular}{cl}
\hline \hline Method and basis set & $Q\left(\mathrm{H}_{2} \mathrm{O}\right)(|e|)$ \\
\hline Ref. 14 & +0.019 \\
Ref. 21 & +0.03 \\
Ref. 22 & +0.035 \\
SZ (present work) & -0.00439 \\
SZP (present work) & -0.00516 \\
DZP (present work) & -0.00089 \\
\hline \hline
\end{tabular}

resulting interactions of water molecule with its periodic images. Besides small splitting of the doubly degenerate nanotube bands near the HOMO level of $\mathrm{H}_{2} \mathrm{O}$ molecule, there is no noticeable change in the nanotube electronic structure. This implies that the charge-transfer-induced doping is not a relevant mechanism of the physisorption of $\mathrm{H}_{2} \mathrm{O}$ molecule.

Quite a few experimental and theoretical studies have considered the effect of humidity or water adsorption on the CNTFET. ${ }^{14,15,21,22} \mathrm{~A}$ few of them claimed the electron transfer from the CNT to the adsorbed $\mathrm{H}_{2} \mathrm{O}$ molecule based on the Mulliken population analysis. However, it is well documented in quantum chemistry textbooks that the Mulliken population analysis should be taken with caution because it is not unique and sensitively depends on the quality of basis sets. ${ }^{25}$ A mere overlap between the tails of the molecular charges could result in a trace of charge transfer in the Mulliken charge analysis. However, if the adsorption states in a semiconductor induce a true charge transfer, it should be accompanied with electron depletion in the valence bands (hole doping) or electron accumulation in the conduction band (electron doping). Simple molecular physisorption may induce a small overlap between the tails of the overall charge densities. This could slightly affect the electronic structure deep in the valence bands, as shown in Fig. 2(b), but should have negligible effect on the electronic structure near the Fermi level.

For explicit demonstration, we calculate the Mulliken charge for the $\mathrm{H}_{2} \mathrm{O}$-adsorbed carbon nanotube at different levels of Gaussian basis sets. We use the SeqQuest program in which the contracted Gaussian basis sets for the corresponding PBE norm-conserving pseudopotentials are optimized for both molecules and bulk solids and thus more balanced than those for the standard quantum chemistry codes. ${ }^{26}$ As noted in Table I, the calculated Mulliken charge transfer within our computational scheme indeed converges to zero as the completeness of the basis set increases, i.e., from the single zeta (SZ) and single zeta polarization (SZP) to double zeta polarization (DZP) level. This obviously indicates that the previously alleged charge transfer should have been cross-checked with variable basis sets.

To explain the humidity-induced hysteresis, instead of the direct charge transfer from the CNT to the neutral water molecule, we note that the actual CNTFET has various components, including metal electrode, metal-CNT interface, and the gate dielectric surface. The effect of water adsorption on such device parts could have substantial effect on the transport properties of the CNTFET. For example, the metal-CNT interface may have an increased chemical activity, and the molecule adsorption on the metal-CNT interface region could result in the modification of the Schottky barrier. 

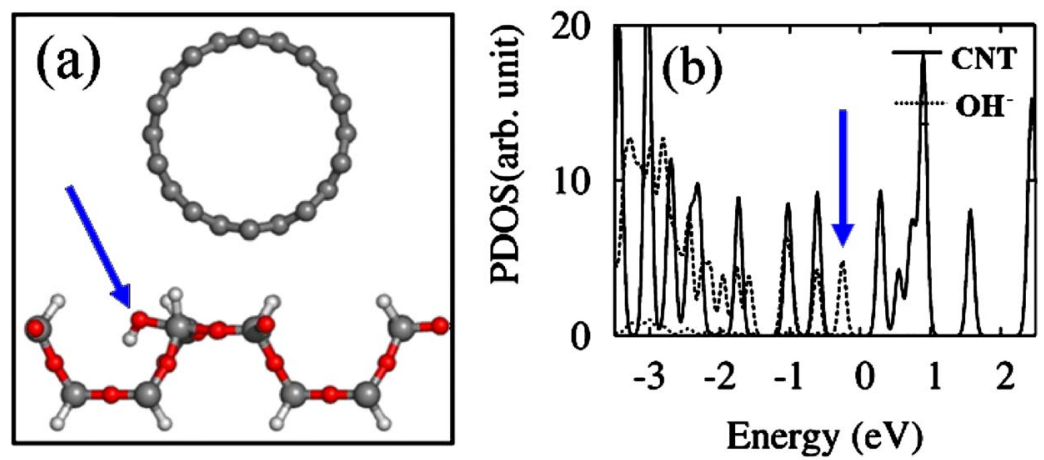

FIG. 3. (Color online) (a) Model for $\mathrm{OH}$ adsorption, indicated by an arrow, on the surface of the gate oxide $\left(\mathrm{SiO}_{2}\right)$ of the CNTFET. (b) The electronic structure for the geometry (a) with an additional electron. The partial densities for the carbon nanotube and $\mathrm{OH}$ are presented by the solid and dotted lines, respectively, with respect to the Fermi level of the CNT.

On the other hand, the accumulation of water layer on the gate oxide surface may help gathering of some ionic substances in the vicinity of the carbon nanotube. In this regard, we note that a previous study discussed the possibility of the concentration of $\mathrm{OH}$ ions on the $\mathrm{SiO}_{2}$ surface. ${ }^{15} \mathrm{In}$ the present work, we investigate the effect of such ionic substances in terms of electronic structures. We first calculate the electronic structure of the $\mathrm{OH}$-adsorbed $\mathrm{SiO}_{2}$ surface near the carbon nanotube. We find that the weakly bonded neutral $\mathrm{OH}$ on the $\mathrm{SiO}_{2}$ surface produces a half-filled molecular level at the Fermi level. Next, considering the same system with one additional electron, we obtain the optimized geometry for the CNT on the $\mathrm{SiO}_{2}$ surface with an adsorbed $\mathrm{OH}^{-}$, as shown in Fig. 3(a). The aforementioned half-filled $\mathrm{OH}$ level is occupied by the additional electron, as indicated by an arrow in Fig. 3(b). We thus conclude that the $\mathrm{OH}$ ions on the gate oxide surface near the carbon nanotube could be electron trap centers under an elevated gate voltage.

In summary, we performed density-functional calculations to investigate the effect of water adsorption on the performance of the CNTFET. We showed that the effect of doping by the simple physisorption of $\mathrm{H}_{2} \mathrm{O}$ molecule is negligible. We noted that the alleged charge transfer between the $\mathrm{H}_{2} \mathrm{O}$ molecule and the nanotube in previous literatures has been based on incorrect interpretation of the Mulliken analysis. We instead suggested that the effect of accumulated ionic substances via the help of water layer on either the gate oxide surface or the metal-CNT interface could be a more convincing scenario to explain the humidity-induced hysteresis.

This work was supported by the Ministry of Information and Communication, Republic of Korea, under Project No. A1100-0501-0073. This research was also supported by grants from KOSEF through the Center for Nanotubes and Nanostructured Composites and by Korea Research Foundation Grant funded by Korea Government (MOEHRD, Basic Research Promotion Fund) (KRF-2005-070-C00054), under which the calculations were performed by using the supercomputing resources of the Korea Institute of Science and Technology Information (KISTI). One of the authors (N.P.) would like to acknowledge the support from KISTI under the Grand Challenge Support Program with S. Lee as the technical supporter.

${ }^{1}$ S. J. Tans, A. R. M. Verschueren, and C. Dekker, Nature (London) 393, 49 (1998).

${ }^{2}$ R. Martel, T. Schmidt, H. R. Shea, T. Hertel, and Ph. Avouris, Appl. Phys. Lett. 73, 2447 (1998).

${ }^{3}$ A. Bachtold, P. Hadley, T. Nakanishi, and C. Dekker, Science 294, 1317 (2001).

${ }^{4}$ C. T. White and T. N. Todorov, Nature (London) 393, 240 (1998).

${ }^{5}$ A. Javey, J. Guo, Q. Wang, M. Lundstrom, and H. Dai, Nature (London) 424, 654 (2003).

${ }^{6}$ J. Kong, N. R. Franklin, C. Zhou, M. G. Chapline, S. Peng, K. Cho, and H. Dai, Science 287, 622 (2000).

${ }^{7}$ S. J. Tans, M. H. Devoret, H. Dai, A. Thess, R. E. Smalley, L. J. Geerligs, and C. Dekker, Nature (London) 386, 474 (1997).

${ }^{8}$ M. S. Fuhrer, J. Nygard, L. Shih, M. Forero, Y. G. Yoon, M. S. C. Mazzoni, H. J. Choi, J. Ihm, S. G. Louie, A. Zettl, and P. L. McEuen, Science 288, 494 (2000).

${ }^{9}$ N. Park and S. Hong, Phys. Rev. B 72, 045408 (2005).

${ }^{10}$ P. G. Collins, K. Bradley, M. Ishigami, and A. Zettl, Science 287, 1801 (2000).

${ }^{11}$ S. H. Jhi, S. G. Louie, and M. L. Cohen, Phys. Rev. Lett. 85, 1710 (2000).

${ }^{12}$ G. U. Sumanasekera, C. K. W. Adu, S. Fang, and P. C. Eklund, Phys. Rev. Lett. 85, 1096 (2000).

${ }^{13}$ A. Zahab, L. Spina, and P. Poncharal, Phys. Rev. B 62, 10000 (2000).

${ }^{14}$ P. Na, H. Kim, H. M. So, K. J. Kong, H. Chang, B. Ryu, Y. Choi, J. O. Lee, B. K. Kim, J. J. Kim, and J. Kim, Appl. Phys. Lett. 87, 093101 (2005).

${ }^{15}$ W. Kim, A. Javey, O. Vermesh, Q. Wang, W. Li, and H. Dai, Nano Lett. 3, 193 (2003).

${ }^{16}$ G. Kresse and J. Hafner, Phys. Rev. B 47, R558 (1993); Phys. Rev. B 49, 14251 (1994).

${ }^{17}$ G. Kresse and J. Furthmüller, Phys. Rev. B 54, 11169 (1996); Comput. Mater. Sci. 6, 15 (1996).

${ }^{18}$ J. P. Perdew, K. Burke, and M. Ernzerhof, Phys. Rev. Lett. 77, 3865 (1996).

${ }^{19}$ D. M. Ceperley and B. J. Alder, Phys. Rev. Lett. 45, 566 (1980).

${ }^{20}$ G. Kresse and D. Joubert, Phys. Rev. B 59, 1758 (1999).

${ }^{21}$ R. Pati, Y. Zhang, and S. K. Nayak, Appl. Phys. Lett. 81, 2638 (2002).

${ }^{22}$ J. Zhao, A. Buldum, J. Han, and J. P. Lu, Nanotechnology 13, 195 (2002).

${ }^{23}$ Y. Zhao and D. G. Truhlar, J. Chem. Theory Comput. 1, 415 (2005).

${ }^{24}$ J. P. Perdew, J. Tao, V. N. Staroverov, and G. E. Scuseria, J. Chem. Phys. 120, 6898 (2004).

${ }^{25}$ F. L. Pilar, Elementary Quantum Chemistry, 2nd ed. (McGraw-Hill, New York, 1990), pp. 379-381.

${ }^{26}$ P. A. Schultz, Sandia National Laboratories, http://dft.sandia.gov/Quest 